\title{
Maternal Stress During Pregnancy Is Associated with Decreased Cortisol and Cortisone Levels in Neonatal Hair
}

\author{
Bibian van der Voorn ${ }^{a} \quad$ Jonneke J. Hollanders ${ }^{a} \quad$ Noera Kieviet $^{b}$ \\ Koert M. Dolman ${ }^{b}$ Yolanda B. de Rijke ${ }^{c}$ Elisabeth F.C. van Rossum ${ }^{d}$ \\ Joost Rotteveel $^{\mathrm{a}}$ Adriaan Honig $^{\mathrm{b}, \mathrm{e}}$ Martijn J.J. Finken ${ }^{\mathrm{a}}$ \\ ${ }^{a}$ Department of Pediatric Endocrinology, VU University Medical Center, Amsterdam, The Netherlands; \\ ${ }^{b}$ Department of Pediatrics, Psychiatry Obstetrics Pediatrics Expert Center, OLVG West, Amsterdam, The Netherlands; \\ 'Department of Clinical Chemistry, Erasmus MC, University Medical Center, Rotterdam, The Netherlands; \\ ${ }^{\mathrm{d}}$ Department of Internal Medicine, Division of Endocrinology, Erasmus MC, University Medical Center, Rotterdam, \\ The Netherlands; ${ }^{e}$ Department of Psychiatry, VU University Medical Center, Amsterdam, The Netherlands
}

\section{Established Facts}

- Maternal stress during pregnancy is associated with unfavorable infant neurodevelopment.

- Hair GC levels offer a retrospective view on chronic GC exposure.

\section{Novel Insights}

- Maternal pre-/postnatal stress was associated with a decrease in neonatal hair glucocorticoid levels postpartum.

- Maternal postnatal stress was associated with an increase in maternal hair glucocorticoid levels postpartum.

- Antidepressant use did not affect neonatal hair glucocorticoid levels.

\section{Keywords}

Early life stress $\cdot$ Stress, psychological · In utero $\cdot$ Infant, newborn · Hair glucocorticoids

\section{Abstract \\ Background: Hair glucocorticoids (GCs) offer a retrospective view on chronic GC exposure. We assessed whether mater-}

nal pre- and postnatal stress was associated with neonatal and maternal hair GCs postpartum (pp). Methods: On the first day pp 172 mother-infant pairs donated hair, of whom 67 had consulted a centre of expertise for psychiatric disorders during pregnancy. Maternal stress was scored on the Hospital Anxiety and Depression Scale during the first/second $(n=46)$, third trimester $(n=57)$, and pp $(n=172)$. Hair cortisol and cortisone levels were determined by liquid chro-

\begin{tabular}{ll}
\hline KARGER & $\begin{array}{l}\text { Ka } 2018 \text { The Author(s) } \\
\text { Published by S. Karger AG, Basel }\end{array}$ \\
E-Mail karger@karger.com & $\begin{array}{l}\text { This article is licensed under the Creative Commons Attribution- } \\
\text { NonCommercial-NoDerivatives 4.0 International License (CC BY- } \\
\text { NC-ND) (http://www.karger.com/Services/OpenAccessLicense). } \\
\text { www.karger.com/hrp }\end{array}$ \\
& $\begin{array}{l}\text { Usage and distribution for commercial purposes as well as any dis- } \\
\text { tribution of modified material requires written permission. }\end{array}$
\end{tabular}

Bibian van der Voorn, $\mathrm{MD}, \mathrm{PhD}$

Department of Pediatric Endocrinology

VU University Medical Center, PO Box 7057

NL-1007 MB Amsterdam (The Netherlands)

E-Mail b.vandervoorn@vumc.nl 
matography-tandem mass spectrometry, and associations with maternal hospital anxiety subscale (HAS) and hospital depression subscale (HDS) scores, and antidepressant use were analyzed with linear regression. Results: Neonatal hair GCs were negatively associated with elevated HAS-scores during the first/second trimester, log $10(\beta[95 \% \mathrm{Cl}])$ cortisol $-0.19(-0.39$ to 0.02$) p=0.07$, cortisone $-0.10(-0.25$ to 0.05$)$ $p=0.17$; third trimester, cortisol $-0.17(-0.33$ to 0.00$) p=0.05$, cortisone $-0.17(-0.28$ to -0.05$) p=0.01$; and pp, cortisol -0.14 ( -0.25 to -0.02$) p=0.02$, cortisone -0.07 ( -0.16 to 0.02 ) $p=0.10$. A similar pattern was observed for elevated HDSscores. Maternal hair GCs were positively associated with elevated HAS-scores pp (cortisol 0.17 [0.01 to 0.32$] p=0.04$, cortisone 0.18 [0.06 to 0.31 ] $p=0.01$ ), but not prenatally or with elevated HDS-scores. Antidepressant use was associated with elevated maternal hair GCs $(p \leq 0.05)$, but not with neonatal hair GCs. Conclusion: Exposure to excessive preand perinatal maternal stress was associated with a decrease in neonatal hair GCs, while elevated stress-scores around birth were associated with increased maternal hair GCs and elevated stress-scores earlier in gestation were not associated with maternal hair GCs pp. Further studies are needed to test associations with infant neurodevelopment.

(c) 2018 The Author(s)

Published by S. Karger AG, Basel

\section{Introduction}

Anxiety or depressive disorders are associated with alterations in hypothalamic-pituitary-adrenal (HPA) axis activity and reactivity, although the evidence is not unequivocal [1-4]. Anxiety and depressive disorders are common in pregnancy, with numbers ranging from 1 in 10 to 1 in 5 pregnant women [5-7]. Although many observational studies described associations between prenatal exposure to maternal stress and neurodevelopmental problems $[8,9]$, caution must be exercised in the interpretation of some of these findings due to the use of subjective measures of stress, while quantitative indices of HPA axis activity are lacking [10].

As part of the physiological changes during pregnancy, both maternal and foetal glucocorticoids (GCs) exert a positive feedback effect on the placenta by stimulating the synthesis of placental corticotropin-releasing hormone $(\mathrm{CRH})$. Due to this physiological feed-forward response, maternal cortisol increases during gestation [11]. At the same time, increasing oestrogen levels augment the synthesis of corticosteroid-binding globulin [12], resulting in only a modest increase in free cortisol [13]. Moreover, placental $11 \beta$-hydroxysteroid dehydrogenase type 2
(11 $\beta$-HSD2) converts maternal cortisol to inert cortisone. Accordingly, the foetus is partially protected from overexposure to maternal cortisol [14]. Lower placental $11 \beta$-HSD 2 activity and, consequently, increased delivery of maternal cortisol to the foetus has been associated with decreased foetal growth [15]. Longer-term consequences of increased foetal exposure to maternal cortisol may include increased HPA axis reactivity and susceptibility to neurodevelopmental problems [8].

Hair cortisol and cortisone levels represent long-term GC exposure in adults and children above the age of 4 years [16-18]. Accordingly, GC levels in newborn hair might offer a retrospective view on the GC regulation during the last part of pregnancy $[19,20]$. Kapoor et al. [21] studied hair GC levels in the offspring of rhesus monkeys that were randomized to receive exposure to a startle paradigm for 10 min per day, 5 days a week for one-fifth the duration of pregnancy, and found decreased hair cortisol in the exposed offspring, but no difference in hair cortisone. In humans, cortisol in neonatal hair obtained directly postpartum (pp) was higher with advancing gestational age and birth weight [22]. Unfortunately, in this study the impact of maternal stress was not studied. A recent study in humans [20], testing neonatal hair GC levels in association with maternal hair GC levels and perceived stress, found similar results as Kapoor et al. [21] did. However, hair cortisone levels were not taken into account, and hair cortisol levels were measured with an immunoassay technique. Moreover, this study had only included physically and mentally healthy mothers, presumably with low amounts of prenatal stress.

Therefore, in the present study we assessed whether pre- and perinatal exposure to maternal stress is associated with neonatal hair cortisol and cortisone levels directly $\mathrm{pp}$. In addition, we tested associations between maternal stress and GCs in maternal hair obtained at the same time. To this end, we used data from a cohort in which women with severe distress during pregnancy were overrepresented.

\section{Methods}

Study Design and Participants

The present study was part of a prospective cohort study that aimed to explore biomarkers, including neonatal hair GCs and 5-hydroxyindoleacetic acid level in urine, for poor neonatal adaptation after prenatal exposure to selective antidepressants (SADs) and maternal stress [23, 24].

A total of 172 mother-infant pairs were recruited at the maternity department, as well as at the psychiatric-obstetric-paediatric (POP) clinic of the OLVG-West Hospital, Amsterdam, the 
Table 1. Characteristics of mother-infant pairs

\begin{tabular}{|c|c|c|c|}
\hline & $\begin{array}{l}\text { Total group } \\
(n=172)\end{array}$ & $\begin{array}{l}\text { POP mothers } \\
(n=67)\end{array}$ & $\begin{array}{l}\text { Other mothers } \\
(n=105)\end{array}$ \\
\hline \multicolumn{4}{|l|}{ Neonatal } \\
\hline Males & $92(53)$ & $34(51)$ & $58(55)$ \\
\hline Gestational age, weeks & $39.4 \pm 1.7$ & $39.2 \pm 1.6$ & $39.5 \pm 1.7$ \\
\hline Birth weight, $g$ & $3,445.5 \pm 582.6$ & $3,358.5 \pm 511.4$ & $3,500.2 \pm 619.3$ \\
\hline Percentile & $53.6 \pm 26.4$ & $49.3 \pm 24.0$ & $56.3 \pm 27.6$ \\
\hline Hair cortisol pp, pg/mg hair & $162.8(102.8-232.2)$ & $155.3(111.4-202.9)$ & $171.3(96.8-291.0)$ \\
\hline Hair cortisone pp, pg/mg hair & $83.2(63.1-109.8)$ & $79.3(63.9-105.1)$ & $87.2(61.4-128.9)$ \\
\hline \multicolumn{4}{|l|}{ Maternal } \\
\hline Primiparous & $85(49)$ & $29(43)$ & $56(53)$ \\
\hline Age, years & $33.8 \pm 4.7$ & $33.7 \pm 4.5$ & $34.0 \pm 4.8$ \\
\hline \multicolumn{4}{|l|}{ Ethnicity } \\
\hline Dutch & $97(56)$ & $41(61)$ & $56(53)$ \\
\hline Caucasian, non-Dutch & $16(9)$ & $4(6)$ & $12(11)$ \\
\hline Non-Caucasian & $59(35)$ & $22(33)$ & $37(35)$ \\
\hline \multicolumn{4}{|l|}{ Antidepressants } \\
\hline SSRI & $45(26)$ & $44(66)$ & $1^{\dagger}(1)$ \\
\hline SNRI & $7(4)$ & $7(10)$ & $--^{\dagger}$ \\
\hline NaSSA & $9(5)$ & $8(12)$ & $1^{\dagger}(1)$ \\
\hline Combination* & $5(3)$ & $5(8)$ & $--^{\dagger}$ \\
\hline \multicolumn{4}{|l|}{ HADS score pp } \\
\hline HAS score $\geq 8$ & $30(17)$ & $19(28)$ & $11^{\dagger}(11)$ \\
\hline HDS score $\geq 8$ & $19(11)$ & $11(16)$ & $8(8)$ \\
\hline Hair cortisol pp, pg/mg hair & $5.4(3.6-10.6)$ & $6.9(4.4-12.0)$ & $4.8^{\dagger}(3.4-9.9)$ \\
\hline Hair cortisone $\mathrm{pp}, \mathrm{pg} / \mathrm{mg}$ hair & $19.5(14.5-31.2)$ & $21.7(15.4-46.9)$ & $18.2^{\dagger}(12.9-26.8)$ \\
\hline
\end{tabular}

Data are presented as mean $\pm \mathrm{SD}$, median (interquartile range), or $n(\%)$. pp, postpartum; HADS, Hospital Anxiety and Depression Scale; HAS, Hospital Anxiety Scale; HDS, Hospital Depression Scale; SSRI, selective serotonin reuptake inhibitors; SNRI, serotonin-norepinephrine reuptake inhibitors, NaSSA, noradrenergic or specific serotonin antidepressants. * These women were treated with a combination of SSRI with NaSSA $(n=4)$, or NaSSA with SNRI $(n=1){ }^{\dagger}$ Different from POP mothers, $p<0.05$.

Netherlands, which offers consultation to women with psychiatric disorders before, during and after pregnancy on an outpatient basis. The reasons for which pregnant women sought advice at the POP clinic were (1) a history of psychiatric disease, and/or (2) symptoms of distress, and/or (3) current or past use of antidepressants. Approximately one-third $(n=65)$ of our sample consisted of women who visited the POP clinic. The other part of the sample

( $n=107)$ consisted of mothers admitted pp to the maternity ward for medical reasons in themselves and/or in their infants. Therefore, in this cohort, women who experienced severe distress during pregnancy were overrepresented. Among participants, $66(38 \%)$ used SADs, including selective serotonin reuptake inhibitors (SSRIs), serotonin-norepinephrine reuptake inhibitors (SNRIs), noradrenergic or specific serotonin antidepressants (NaSSAs), or a combination of these. Sixty-four of these women sought advice at the POP clinic.

Inclusion and exclusion criteria were similar for both groups. Inclusion criteria were an expected hospital stay of $\geq 72 \mathrm{~h}$ after delivery, and willingness to donate hair from themselves and their infants, and to complete the Hospital Anxiety and Depres- sion Scale (HADS) questionnaire directly pp. Exclusion criteria were use of psychotropic medication other than SADs, use of systemic corticosteroids, non-pharmacologic drugs, or alcohol, smoking during the third trimester of pregnancy, insufficient knowledge of the Dutch or English language, mental impairment of one or both parents and multiple pregnancies. Parents were informed and written informed consent was obtained within $24 \mathrm{~h}$ after delivery. The study was approved by the Medical Ethics Committees of the OLVG-West Hospital and the VU University Medical Center.

\section{Assessment of Maternal Stress}

As part of standard care at the POP clinic, the HADS [25] was administered as an index of stress experienced in the previous week. The HADS contains 14 items, namely, 7 for anxiety and 7 for depression. From these items, Hospital Anxiety Subscale (HAS) and Hospital Depression Subscale (HDS) scores are derived. A score $\geq 8$ (out of 21 points for each subscale separately) is considered the cut-off for relevant stress $[25,26]$. The retest reliability of the HADS was found to correlate well with the previous 6 weeks [26]. Furthermore, among pregnant women, the anxiety 


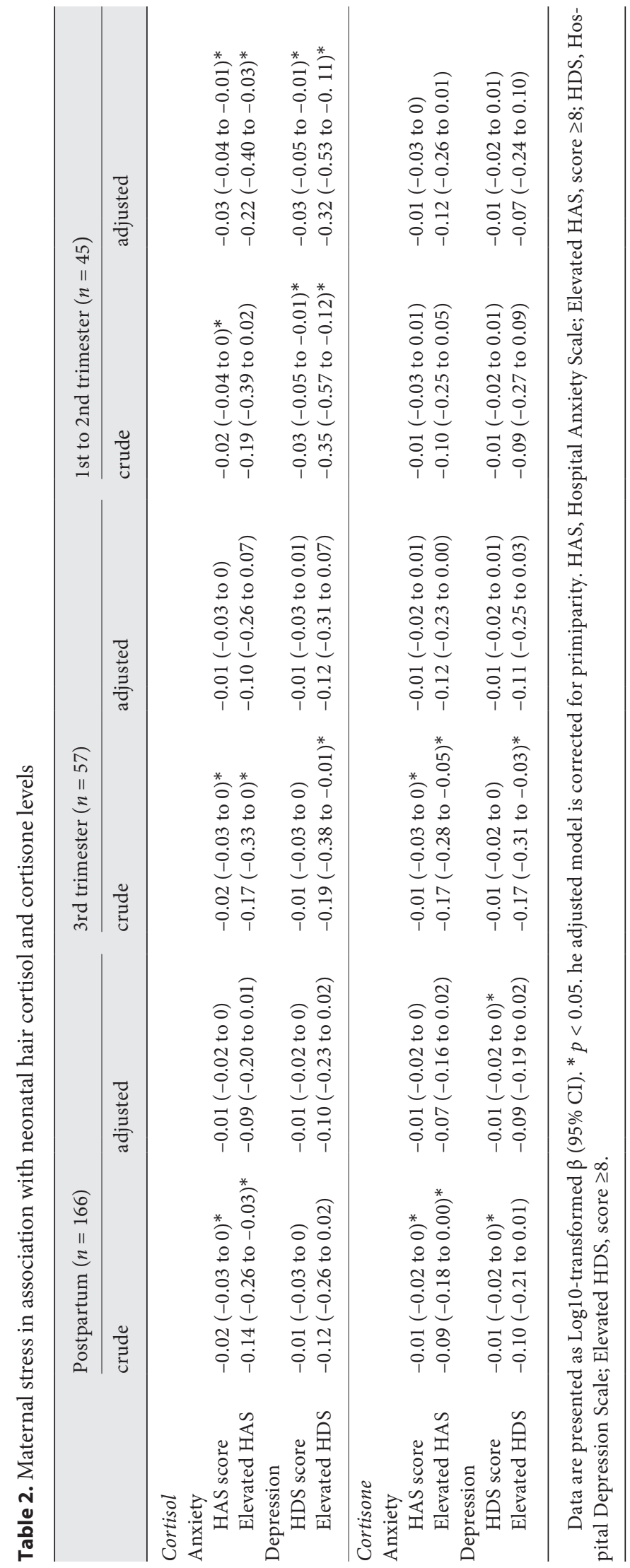

subscales of the HADS and the Edinburgh Postnatal Depression Scale showed strong correlation [27].

During the first or second trimester, and/or the third trimester, the HADS was administered only in the women who visited the POP clinic. Directly pp, that is, within $12-36 \mathrm{~h}$, the HADS was administered in all women. At the maternity ward, mothers were asked whether they had used SADs at least during the last 2 weeks of pregnancy.

\section{Hair Glucocorticoid Levels}

Mother-infant pairs donated hair on the first day pp. A lock of hair was cut from the posterior vertex as close as possible to the scalp. A minimum of $1.25 \mathrm{mg}$ hair is needed for a reliable measurement. Foetal hair growth velocity and the timing of transition from lanugo via vellus into terminal hair strands vary significantly between infants [28]. Therefore, the total length of neonatal hair was analyzed. In mothers, the centimeter of hair closest to the scalp was analyzed, representing the mean levels of cortisol and cortisone during the last month of gestation, as adult hair grows approximately 1 (range 0.6-1.4) cm per month [29]. There is evidence of $11 \beta$-HSD2 expression in human eccrine sweat glands and vascular endothelium [30], raising the possibility of local conversion of blood-borne cortisol to cortisone within skin and/or hair follicles. Therefore, it is unknown which analyte is the best representative of serum cortisol. The sum of hair GCs might be indicative of chronic circulating cortisol and was therefore calculated.

Hair cortisol and cortisone levels were measured as described previously by Noppe et al. [17]. In short, hair was washed with isopropanol, and hair GCs were extracted using methanol and solidphase extraction. Subsequently, cortisol and cortisone concentrations were quantified by liquid chromatography-tandem mass spectrometry (Waters XEVO-TQ-S system, Waters Corporation, Milford, MA, USA) with positive electrospray ionization, and reported in $\mathrm{pg} / \mathrm{mg}$ hair. The Lower Limit of Quantitation of our assay is dependent on the amount of hair extracted. An intra-assay CV of $8.9 \%$ was measured at a hair cortisol concentration of $1.8 \mathrm{pg} / \mathrm{mg}$. The intra-assay CV for hair cortisone was $4.4 \%$ at a level of 12.5 $\mathrm{pg} / \mathrm{mg}$.

\section{Data Analysis}

Maternal and neonatal characteristics were compared between the pairs whose mothers visited the POP clinic and the pairs admitted for medical reasons, using independent $t$ tests, chi square, or Fisher exact tests (Table 1).

Hair cortisol and cortisone levels were skewed to the right and therefore logarithmically transformed prior to analysis. Linear regression was used to assess associations between HADS scores and hair GC levels. Associations with maternal stress were assessed with hair GC level as dependent factor, and HAS or HDS score as continuous or dichotomous (with a score of $\geq 8$ points as cut-off for elevated stress) independent factor [25]. Among infants whose mothers visited the POP clinic, the relative contributions of preand perinatal stress exposure were tested by using combinations of (1) low prenatal and low perinatal (reference), (2) low prenatal and high perinatal, (3) high prenatal and low perinatal, and (4) high prenatal and high perinatal levels of stress exposure. Low prenatal stress exposure was defined as low HAS and HDS scores in both the first/second and the third trimester, while high prenatal stress exposure was defined as a score $\geq 8$ on one or both subscales in the first/second and/or the third trimester. Likewise, low perinatal 
Table 3. Maternal stress over time among the women who visited the POP clinic, in association with neonatal hair cortisol and cortisone levels

\begin{tabular}{|c|c|c|c|c|c|}
\hline Exposure category & $n$ & \multicolumn{2}{|c|}{ Cortisol } & \multicolumn{2}{|c|}{ Cortisone } \\
\hline Low prenatally and low perinatally & 36 & Ref. & & Ref. & \\
\hline High prenatally and low perinatally & 11 & -0.09 & -0.30 to 0.12 & -0.14 & -0.29 to 0.02 \\
\hline Low prenatally and high perinatally & 6 & -0.03 & -0.29 to 0.23 & 0.01 & -0.18 to 0.20 \\
\hline Low prenatally and low perinatally & 50 & Ref. & & Ref. & \\
\hline High prenatally and low perinatally & 2 & -0.09 & -0.36 to 0.19 & -0.18 & -0.38 to 0.03 \\
\hline Low prenatally and high perinatally & 5 & -0.13 & -0.55 to 0.29 & -0.14 & -0.45 to 0.17 \\
\hline High prenatally and high perinatally & 8 & -0.27 & -0.49 to $-0.05^{*}$ & -0.17 & -0.33 to $0.00^{*}$ \\
\hline
\end{tabular}

stress exposure was defined as low HAS and HDS scores pp, while high perinatal stress exposure was defined as a score $\geq 8$ on one or both subscales pp. Associations with maternal SAD use were analyzed with hair GC level as dependent factor, and SAD use as dichotomous independent factor.

Confounders were selected a priori, based on the literature [21$23,31]$. Sex, birth weight percentile, gestational age and primiparity were added to the multivariable model, one by one. Subsequently, based on statistical impact (i.e., a $>10 \%$ change in beta) the final model was created. When a confounder was found to have a statistical impact on more than $50 \%$ of the associations being analyzed, we also explored the univariate association with the outcome. In addition, similar to Kapoor et al. [21], interaction between perinatal stress (HADS scores pp) and sex on neonatal hair GC levels was tested.

\section{Results}

The characteristics of participants are shown in Table 1. A total of 67 women visited the POP clinic, of whom $98 \%$ reported SAD use, $28 \%$ had an elevated HAS score and $16 \%$ had an elevated HDS score. For mothers admitted pp for medical reasons in themselves and/or in their infants $(n=105)$, these numbers $(2,11$ and $8 \%$ respectively) were similar to previously reported prevalence rates in the normal population [5-7, 32]. Sex distribution, gestational age, birth weight, parity, maternal age and ethnicity did not differ between the groups. Neonatal hair cortisone levels were significantly lower in female neonates (median [IQR] 75.1 [59.8-99.7] pg/mg for females and 92.1 [65.4-129.2] pg/mg for males, $p=0.049)$. Neo- natal hair cortisol levels did not differ significantly between the sexes.

The characteristics of mother-infant pairs by time point are shown in online supplementary Table 1 (see www.karger.com/doi/10.1159/000495007 for all online suppl. material). The great majority of women who visited the POP clinic during the first or second, and/or the third trimester, used SADs: 44 out of 46 (96\%) and 54 out of 57 (95\%) respectively. Those who visited the POP clinic during the first or second, and/or third trimester, more often used SADs during the entire pregnancy: 41 out of $46(89 \%)$ and 43 out of $57(75 \%)$ respectively.

\section{The Association Between Maternal Stress and \\ Neonatal Hair GCs}

HADS scores during pregnancy were only known for the mothers who visited the POP clinic $(n=65)$, namely, 46 during the first or second trimester, and 57 during the third trimester. As part of the routine follow-up, 38 of them were seen on both occasions -8 only during the first or second trimester and 19 only during the third trimester (online suppl. Fig. 1).

We were able to collect enough hair (median [IQR] 5.1 [3.2-8.9] $\mathrm{mg}$, while at least $1.25 \mathrm{mg}$ is required) in 166 newborns (97\%). In crude analyses, maternal anxiety and depression experienced during pregnancy or pp were negatively associated with the neonatal hair cortisol level (Table 2). Similar, but weaker, associations were found for the neonatal hair cortisone level. Associations with the sum of neonatal hair GCs resembled those with neonatal 


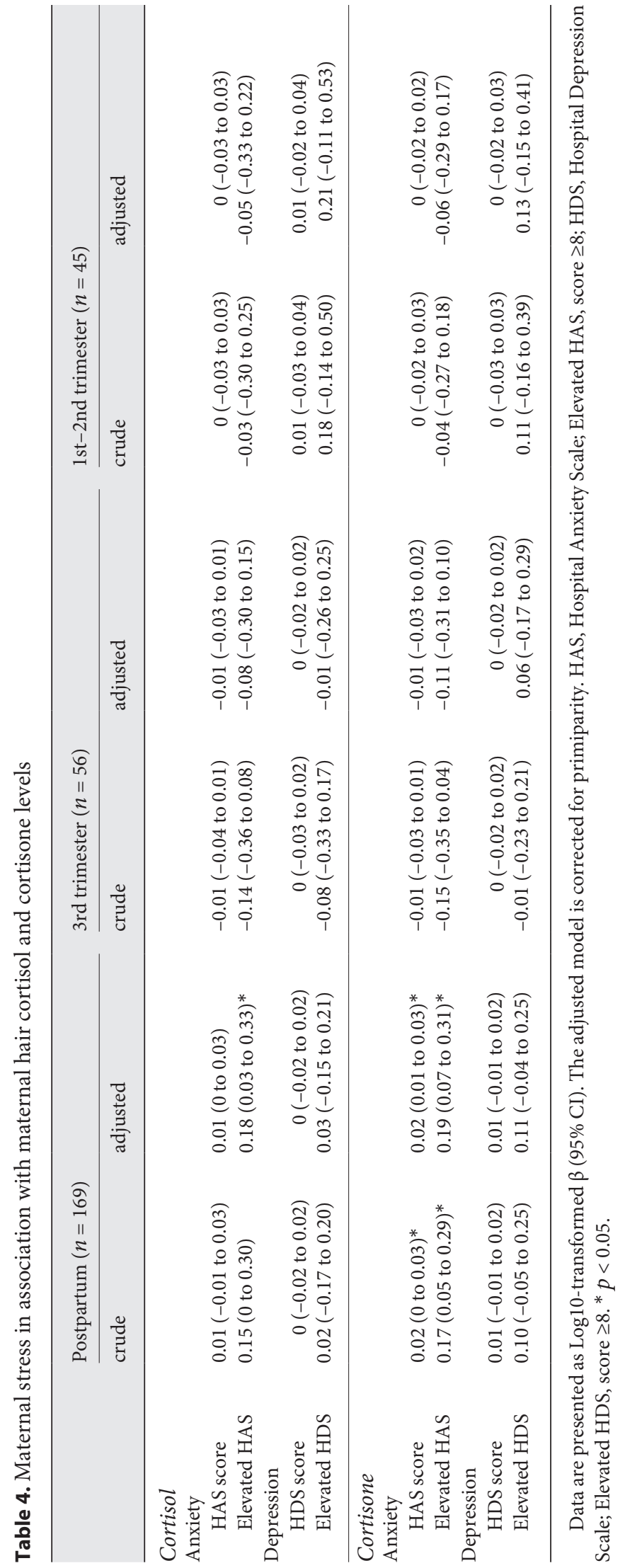

hair cortisol alone (data not shown). Correction for sex, gestational age, or birth weight percentile did not change these associations. Correction for primiparity strengthened the associations with first or second trimester stress scores. Associations with maternal stress during the third trimester or pp became non-significant or disappeared when parity was factored in (Table 2). When tested univariately, primiparity was associated with higher neonatal hair cortisol and cortisone levels ( $\log 10$-transformed $\beta$ [95\% CI]: $0.24[0.16 ; 0.32] p<0.001$, and 0.10 [0.04; 0.17] $p<0.001$, respectively). There was no evidence for interaction between dichotomous HADS, HAS or HDS scores and sex on neonatal hair GC levels.

Among the infants whose mothers visited the POP clinic, persistent exposure to elevated maternal stress was associated with the largest decrease in neonatal hair GC levels (Table 3).

\section{The Association between Maternal Stress and Maternal Hair GCs}

Maternal anxiety experienced directly pp, but not in gestation, was positively associated with maternal hair cortisol and cortisone levels pp (Table 4). Similar findings were obtained with the sum of maternal hair GCs (data not shown). Correction for sex, gestational age, birth weight percentile or primiparity did not change these associations.

\section{The Association between SAD Use and Neonatal and Maternal Hair GC Levels}

Sixty-six women (38\%) used SADs. Forty-five women were on SSRIs, 7 on SNRIs, 9 on NaSSAs and 5 on a combination of these, including SSRIs with NaSSAs $(n=4)$ and NaSSAs with SNRIs $(n=1)$.

The use of SADs was not associated with neonatal hair GC levels, but it was positively associated with maternal hair cortisol and cortisone levels pp (log-10 transformed $\beta[95 \% \mathrm{CI}]: 0.14[0.02,0.26] p=0.02$, and $0.18[0.09 ; 0.27]$ $p<0.001$ respectively).

\section{Discussion}

In this study, maternal stress during pregnancy and $\mathrm{pp}$ was associated with decreased neonatal hair GC levels, with the lowest values seen in the infants of mothers with persistent distress. In addition, maternal stress pp, but not in gestation, was associated with increased maternal hair GC levels pp. SAD use during pregnancy was unlikely to explain these associations. 
We recently published data suggesting that neonatal hair GC levels are influenced by the third-trimester increase in HPA axis activity [19]. This phenomenon might offer an explanation for the high neonatal hair GC levels observed in our study, although evidence is lacking concerning which part of intrauterine GC regulation is reflected in neonatal hair. It has been hypothesized that neonatal hair GC levels might reflect amniotic-fluid GC levels [21]. However, this must be balanced against evidence from studies in adults suggesting that hair GCs reflect the body's HPA axis activity, with hair GCs being associated with long-term integrated salivary cortisol [33]. It is conceivable that neonatal hair GCs reflect a combination of maternal and foetal GCs, determined by placental factors like the third-trimester increase in placental CRH and $11 \beta$-HSD2 activity and foetal adrenal maturation. The balance between cortisol and cortisone in hair may be different than in blood, as $11 \beta$-HSD2 is widely expressed in epithelial tissues [30].

It is unclear why the HAS score, but not the HDS score pp, was positively associated with maternal hair GCs pp. In general, in patients with psychiatric symptomatology anxiety and depressive symptoms are highly overlapping. Findings from studies investigating associations between HADS scores and indices of HPA axis activity in matrices other than hair, like saliva, are highly contradictory. One study found that the HAS score, but not the HDS score, correlated positively with the cortisol awakening response among patients with coronary artery disease [34]. Another study among patients with coronary artery disease found that the HDS score correlated positively with salivary cortisol in men, but not in women, while in women, but not in men, the HAS score correlated negatively with salivary cortisol [35]. In patients with low back pain, HADS scores were unrelated to the diurnal cortisol rhythm measured in saliva [36], while in the normal population, HADS scores were inversely associated with peak salivary cortisol during a psychological stress protocol [37].

Previous studies have shown that maternal stress is associated with reduced placental $11 \beta$-HSD2 activity [3841 , thereby allowing a larger proportion of maternal cortisol to reach the foetus. In contrast, we found that higher maternal stress scores were associated with lower neonatal hair GCs levels, in spite of evidence of increased maternal HPA axis activity. There are several explanations possible for these discrepant findings. First, maternal HPA axis activity may be lower in those pregnant women with chronic stress, so that, despite reduced placental $11 \beta$-HSD2 activity, a smaller amount of cortisol is able to cross the placenta. Some studies have shown blunted HPA axis activity

Maternal Prenatal Stress and Neonatal Hair GCs in chronic stress [4], but it is unclear whether their findings could be extrapolated to pregnancy, when HPA activity is regulated to a large extent by placental CRH. However, the stressed women in our cohort had elevated hair GC levels, representing increased HPA axis activity in the last trimester. Second, the third-trimester surge in the production of GCs by the foetal adrenal probably contributes much more to the foetal GC pool than maternal HPA axis activity, at least in the last part of gestation. Still, the decrease in placental $11 \beta$-HSD2 activity as observed in chronic distress could contribute significantly to the foetal GC pool at earlier stages of gestation, when placental $11 \beta$-HSD2 activity might be lower [42]. We speculate that increased exposure to maternal cortisol earlier in gestation might lead to a long-lasting suppression of foetal HPA axis activity. Analysis of the entire neonatal hair, which we did in our study, probably reflects the endocrine milieu during the last trimester, as it may take 20 weeks until the scalp is fully covered with anagen phase hair, turning into the catagen phase between week 24 and 28 [43].

In rat pups, GC receptor density in the forebrain was found to develop in a sex-specific manner, with females having more GC receptors than males [44]. It could therefore be hypothesized that prenatal maternal stress might have sex-specific effects on neonatal HPA axis activity. However, we as well as others did not find evidence for sex differences in, or sex-specificity of the effects of prenatal maternal stress on, neonatal hair GC levels [20-22].

Foetal exposure to maternal stress or to excessive maternal GCs has been associated with neurodevelopmental problems as well as alterations in HPA axis settings [8,9]. The mechanisms behind these associations are not yet fully elucidated. Therefore, long-term follow-up of our cohort is warranted to explore associations with HPA axis development, including aspects like stress reactivity and development of diurnal rhythmicity, in addition to neurodevelopment [12].

The major strength of this study is the unique sample of women experiencing a wide range of stress levels during pregnancy and pp, including an overrepresentation of severely distressed women. In addition, to the best of our knowledge, this is the first translational study that measured hair GC levels in both infants and their mothers who experienced a reliably quantified amount of stress during pregnancy and pp [45]. A limitation of our study is that HADS scores during pregnancy were known only for the women who sought consultation at the POP clinic. Other limitations are the lack of information on neonatal hair growth (and, thereby, the lack of standardization on newborn hair length), and the self-report of SAD 
use. In addition, the small sample size did not allow us to test associations by type of SAD. Lastly, ideally other tests of HPA axis activity, relating to HPA axis reactivity and rhythmicity, should have been included too [46].

In conclusion, we found that maternal stress was associated with increased maternal hair GC levels and decreased neonatal hair GC levels, with the lowest values seen in children of mothers with persistent distress throughout pregnancy. It is suggested that maternal stress during pregnancy increased intra-uterine GC exposure, thereby suppressing foetal HPA axis activity.

\section{Disclosure Statement}

The authors have no conflicts of interest to disclose.

\section{Funding Source}

This study was financially supported by Nuts Ohra by a nonrestricted grant (SSRI-158115/754). Nuts Ohra was not involved in the design and conduct of the study, collection, management, analysis, and interpretation of the data, neither in preparation, review, or approval of the manuscript and decision to submit the manuscript for publication. The authors report no financial or other relationship relevant to the subject of this article.

\section{Acknowledgements}

Mrs. Kristien Dorst is acknowledged for her great contribution to the hair analysis.

\section{References}

1 Knorr U, Vinberg M, Kessing LV, Wetterslev J. Salivary cortisol in depressed patients versus control persons: a systematic review and meta-analysis. Psychoneuroendocrinology. 2010 Oct;35(9):1275-86.

2 Lewis EJ, Yoon KL, Joormann J. Emotion regulation and biological stress responding: associations with worry, rumination, and reappraisal. Cogn Emotion. 2018 Nov;32(7): 1487-98.

3 Vreeburg SA, Zitman FG, van Pelt J, Derijk RH, Verhagen JC, van Dyck R, et al. Salivary cortisol levels in persons with and without different anxiety disorders. Psychosom Med. 2010 May;72(4):340-7.

4 Zorn JV, Schür RR, Boks MP, Kahn RS, Joëls $\mathrm{M}$, Vinkers $\mathrm{CH}$. Cortisol stress reactivity across psychiatric disorders: A systematic review and meta-analysis. Psychoneuroendocrinology. 2017 Mar;77:25-36.

5 Austin MP, Hadzi-Pavlovic D, Priest SR, Reilly N, Wilhelm K, Saint K, et al. Depressive and anxiety disorders in the postpartum period: how prevalent are they and can we improve their detection? Arch Women Ment Health. 2010 Oct;13(5):395-401.

6 Dennis CL, Falah-Hassani K, Shiri R. Prevalence of antenatal and postnatal anxiety: systematic review and meta-analysis. Br J Psychiatry. 2017 May;210(5):315-23.

7 Shakeel N, Eberhard-Gran M, Sletner L, Slinning K, Martinsen EW, Holme I, et al. A prospective cohort study of depression in pregnancy, prevalence and risk factors in a multi-ethnic population. BMC Pregnancy Childbirth. 2015 Jan;15(1):5.
8 Duthie L, Reynolds RM. Changes in the maternal hypothalamic-pituitary-adrenal axis in pregnancy and postpartum: influences on maternal and fetal outcomes. Neuroendocrinology. 2013;98(2):106-15.

9 Sandman CA, Davis EP, Buss C, Glynn LM. Exposure to prenatal psychobiological stress exerts programming influences on the mother and her fetus. Neuroendocrinology. 2012; 95(1):7-21.

10 Talge NM, Neal C, Glover V; Early Stress, Translational Research and Prevention Science Network: Fetal and Neonatal Experience on Child and Adolescent Mental Health. Antenatal maternal stress and long-term effects on child neurodevelopment: how and why? J Child Psychol Psychiatry. 2007 Mar-Apr; 48(3-4):245-61.

11 Glynn LM, Davis EP, Sandman CA. New insights into the role of perinatal HPA-axis dysregulation in postpartum depression. Neuropeptides. 2013 Dec;47(6):363-70.

12 Trainer PJ. Corticosteroids and pregnancy. Semin Reprod Med. 2002 Nov;20(4):375-80.

13 Demey-Ponsart E, Foidart JM, Sulon J, Sodoyez JC. Serum CBG, free and total cortisol and circadian patterns of adrenal function in normal pregnancy. J Steroid Biochem. 1982 Feb;16(2):165-9.

14 Watterberg KL. Adrenocortical function and dysfunction in the fetus and neonate. Semin Neonatol. 2004 Feb;9(1):13-21.

15 Kajantie E, Dunkel L, Turpeinen U, Stenman UH, Wood PJ, Nuutila M, et al. Placental 11 beta-hydroxysteroid dehydrogenase- 2 and fetal cortisol/cortisone shuttle in small preterm infants. J Clin Endocrinol Metab. 2003 Jan; 88(1):493-500.
16 D’Anna-Hernandez KL, Ross RG, Natvig CL, Laudenslager ML. Hair cortisol levels as a retrospective marker of hypothalamic-pituitary axis activity throughout pregnancy: comparison to salivary cortisol. Physiol Behav. 2011 Aug; 104(2):348-53.

17 Noppe G, de Rijke YB, Dorst K, van den Akker EL, van Rossum EF. LC-MS/MS-based method for long-term steroid profiling in human scalp hair. Clin Endocrinol (Oxf). 2015 Aug;83(2):162-6.

18 Noppe G, Van Rossum EF, Koper JW, Manenschijn L, Bruining GJ, de Rijke YB, et al. Validation and reference ranges of hair cortisol measurement in healthy children. Horm Res Paediatr. 2014;82(2):97-102.

19 Hollanders JJ, van der Voorn B, Kieviet N, Dolman KM, de Rijke YB, van den Akker EL, et al. Interpretation of glucocorticoids in neonatal hair: a reflection of intrauterine glucocorticoid regulation? Endocr Connect. 2017 Nov;6(8):692-9.

20 Romero-Gonzalez B, Caparros-Gonzalez RA, Gonzalez-Perez R, Delgado-Puertas P, Peralta-Ramirez MI. Newborn infants' hair cortisol levels reflect chronic maternal stress during pregnancy. PLoS One. 2018 Jul;13(7): e0200279.

21 Kapoor A, Lubach GR, Ziegler TE, Coe CL. Hormone levels in neonatal hair reflect prior maternal stress exposure during pregnancy. Psychoneuroendocrinology. 2016 Apr;66: $111-7$. 
22 Hoffman MC, D’Anna-Hernandez K, Benitez P, Ross RG, Laudenslager ML. Cortisol during human fetal life: characterization of a method for processing small quantities of newborn hair from 26 to 42 weeks gestation. Dev Psychobiol. 2017 Jan;59(1):123-7.

23 Kieviet N, de Groot S, Noppe G, de Rijke YB, van Rossum EF, van den Akker EL, et al. Is poor neonatal adaptation after exposure to antidepressant medication related to fetal cortisol levels? An explorative study. Early Hum Dev. 2016 Jul;98:37-43.

24 Kieviet N, van Keulen V, van de Ven PM, Dolman KM, Deckers M, Honig A. Serotonin and poor neonatal adaptation after antidepressant exposure in utero. Acta Neuropsychiatr. 2017 Feb;29(1):43-53.

25 Zigmond AS, Snaith RP. The hospital anxiety and depression scale. Acta Psychiatr Scand. 1983 Jun;67(6):361-70.

26 Herrmann C. International experiences with the Hospital Anxiety and Depression Scale-a review of validation data and clinical results. J Psychosom Res. 1997 Jan;42(1):17-41.

27 Matthey S, Valenti B, Souter K, Ross-Hamid C. Comparison of four self-report measures and a generic mood question to screen for anxiety during pregnancy in English-speaking women. J Affect Disord. 2013 Jun;148(23):347-51.

28 Furdon SA, Clark DA. Scalp hair characteristics in the newborn infant. Adv Neonatal Care. 2003 Dec;3(6):286-96.

29 Pragst F, Balikova MA. State of the art in hair analysis for detection of drug and alcohol abuse. Clin Chim Acta. 2006 Aug;370(1-2): 17-49.

30 Smith RE, Maguire JA, Stein-Oakley AN, Sasano H, Takahashi K, Fukushima K, et al. Localization of 11 beta-hydroxysteroid dehydrogenase type II in human epithelial tissues. J Clin Endocrinol Metab. 1996 Sep;81(9): $3244-8$
31 Dettmer AM, Rosenberg KL, Suomi SJ, Meyer JS, Novak MA. Associations between Parity, Hair Hormone Profiles during Pregnancy and Lactation, and Infant Development in Rhesus Monkeys (Macaca mulatta). PLoS One. 2015 Jul;10(7):e0131692.

32 Meunier MR, Bennett IM, Coco AS. Use of antidepressant medication in the United States during pregnancy, 2002-2010. Psychiatr Serv. 2013 Nov;64(11):1157-60.

33 Short SJ, Stalder T, Marceau K, Entringer S, Moog NK, Shirtcliff EA, et al. Correspondence between hair cortisol concentrations and 30-day integrated daily salivary and weekly urinary cortisol measures. Psychoneuroendocrinology. 2016 Sep;71:12-8.

34 Merswolken M, Deter HC, Siebenhuener S, Orth-Gomér K, Weber CS. Anxiety as predictor of the cortisol awakening response in patients with coronary heart disease. Int J Behav Med. 2013 Sep;20(3):461-7.

35 Norris CM, Ljubsa A, Hegadoren KM. Gender as a determinant of responses to a selfscreening questionnaire on anxiety and depression by patients with coronary artery disease. Gend Med. 2009 Sep;6(3):479-87.

36 Harris A, Endresen Reme S, Tangen T, Hansen AM, Helene Garde A, Eriksen HR. Diurnal cortisol rhythm: associated with anxiety and depression, or just an indication of lack of energy? Psychiatry Res. 2015 Aug;228(2): 209-15.

37 de Rooij SR, Schene AH, Phillips DI, Roseboom TJ. Depression and anxiety: associations with biological and perceived stress reactivity to a psychological stress protocol in a middle-aged population. Psychoneuroendocrinology. $2010 \mathrm{Jul} ; 35(6): 866-77$.

38 O’Donnell KJ, Bugge Jensen A, Freeman L, Khalife N, O'Connor TG, Glover V. Maternal prenatal anxiety and downregulation of placental 11 $\beta$-HSD2. Psychoneuroendocrinology. 2012 Jun;37(6):818-26.
39 Seth S, Lewis AJ, Saffery R, Lappas M, Galbally M. Maternal Prenatal Mental Health and Placental $11 \beta-H S D 2$ Gene Expression: Initial Findings from the Mercy Pregnancy and Emotional Wellbeing Study. Int J Mol Sci. 2015 Nov; $16(11): 27482-96$

40 Togher KL, Togher KL, O'Keeffe MM, O'Keeffe MM, Khashan AS, Khashan AS, et al. Epigenetic regulation of the placental HSD11B2 barrier and its role as a critical regulator of fetal development. Epigenetics. 2014 Jun;9(6):816-22.

41 Togher KL, Treacy E, O'Keeffe GW, Kenny LC. Maternal distress in late pregnancy alters obstetric outcomes and the expression of genes important for placental glucocorticoid signalling. Psychiatry Res. 2017 Sep;255:1726.

42 Schoof E, Girstl M, Frobenius W, Kirschbaum M, Repp R, Knerr I, et al. Course of placental 11beta-hydroxysteroid dehydrogenase type 2 and 15-hydroxyprostaglandin dehydrogenase mRNA expression during human gestation. Eur J Endocrinol. 2001 Aug;145(2):18792.

43 Gareri J, Koren G. Prenatal hair development: implications for drug exposure determination. Forensic Sci Int. 2010 Mar;196(1-3):2731.

44 Slotkin TA, Seidler FJ, Wood CR, Lau C. Development of glucocorticoid receptor regulation in the rat forebrain: implications for adverse effects of glucocorticoids in preterm infants. Brain Res Bull. 2008 Jul;76(5):531-5.

45 Liu CH, Snidman N, Leonard A, Meyer J, Tronick E. Intra-individual stability and developmental change in hair cortisol among postpartum mothers and infants: implications for understanding chronic stress. Dev Psychobiol. 2016 May;58(4):509-18.

46 Flom M, St John AM, Meyer JS, Tarullo AR. Infant hair cortisol: associations with salivary cortisol and environmental context. Dev Psychobiol. 2017 Jan;59(1):26-38. 